\title{
Evaluation of Multiusers' Interference on Radiolocation in CDMA Cellular Networks
}

\author{
A. J. Bamisaye, M. O. Kolawole, V. S. A. Adeloye \\ Department of Electrical and Electronics Engineering, The Federal University of Technology, Akure, Nigeria \\ E-mail: \{ayobamisaye, kolawolm, vadeloye\}@yahoo.com \\ Received October 17, 2009; revised November 15, 2009; accepted December 16, 2009
}

\begin{abstract}
Radiolocation has been previously studied for CDMA networks, the effect of Multiple Access Interference has been ignored. In this paper we investigate the problem of Radiolocation in the presence of Multiple Access Interference. An extensive simulation technique was developed, which measures the error in location estimation for different network and user configurations. We include the effects of lognormal shadow and Rayleigh fading. Results that illustrate the effects of varying shadowing losses, number of base stations involved in position location, early-late discriminator offset and cell sizes in conjunction with the varying number of users per cell on the accuracy of radiolocation estimation was presented.
\end{abstract}

Keywords: Code Division Multiple Access, Radiolocation, Multiple Access Interference, Base Station, Mobile Station.

\section{Introduction}

Radiolocation involves: a) identifying the base station (BS) that would participate in the process of subscriber location by selecting a set of BSs within the coverage area that receives intelligible levels of signal from the mobile station (MS) under consideration. b) estimating one-dimensional position which involves each BS, participating in the process, independently producing an estimate of the subscriber location based on its measurements; c) location estimation, that is, estimates from all the participating BSs are used by position location algorithms to produce an accurate estimate of the subscriber location within the coverage area. But, the estimates produced are not always very accurate. The major sources of error in subscriber location systems are: multipath propagation, non-line-of-sight (NLOS), and multiple access interference (MAI). In the case of multipath propagation, accuracy is greatly affected when the reflected rays arrive within a very small period of the first arriving ray. Case is even more worsened when the power of reflected rays is more than the first arriving ray [1]. Several methods have been developed to mitigate the effects of multipath on radiolocation. Typically propagation in wireless communications accrues up to an average of $400-700 \mathrm{~m}[2,3]$ and biases the estimations. By using the a prior information about range error statistics, range estimations made over a period of time and cor- rupted by NLOS errors can be adjusted to near their correct values. An alternative approach is to reduce the weights of the BSs prone to NLOS reception while estimating location using position location algorithms $[4,5]$. Co-channel interference is a problem faced by all the cellular systems. In Code Division Multiple Access (CDMA) networks, users share the same frequency band, but use unique pseudo-noise (PN) codes. Near far effects in CDMA networks are the biggest source of errors in position estimation. Multiple cellular users who are using the same frequency allocation at the same time cause MAI: it greatly affects the performance of Time of Arrival (ToA) estimation of CDMA systems. In CDMA cellular systems, the MSs are power controlled to combat the near-far effect. Thus, for a CDMA network, time based approach is the most promising technique. This paper investigates the effect of MAI and the accuracy of Radiolocation in CDMA cellular network.

This paper models the intracellular and intercellular multiple access interference (MAI) in Section 2, simulation results are presented in Section 3, and finally in Section 4 , the conclusion drawn from the study is summarised.

\section{System Model}

In a CDMA system all the users share the same frequency band. As a result, at a CDMA receiver signals from users, 
other than the intended user, act as interfering signals, thereby giving rise to multiple access interference. Figure 1 represents such a situation.

With reference to Figure 1, the coverage area comprises of cells, $C_{i}, C_{j}, \cdots \cdots$ and $C_{p}$. These cells have $n_{i}, \quad n_{j} \quad n_{k}, \cdots$ and $C_{p}$ users respectively. The BS's $i$, $j, k, \ldots$. and $p$ exercise power control over the users they serve. Let $A_{i}, A_{j}, A_{k} \ldots$ and $A_{p}$ denote their areas. Only few cells have been considered for simplicity of explanation.

\subsection{Modeling Intracellular Multiple Access Interference}

In a CDMA system using binary signaling, the radio signal from the $\mathrm{k}^{\mathrm{th}}$ user, arriving at the BS is given by:

$$
S_{k}\left(t-\tau_{k}\right)=\sqrt{2 P_{k}} c_{k}\left(t-\tau_{k}\right) b_{k}\left(t-\tau_{k}\right) \cos \left(\omega_{c} t+\varphi_{k}\right)
$$

where, $P_{k}$ is the power received from the $\mathrm{k}^{\text {th }}$ user at the BS. Assuming, perfect power control is exercised, we can replace $P_{k}$ by $P$, where $P$ represents the nominal power received at the BS from a user under its power control.

$c_{k}(t)$ is the spreading (or chip) sequence for a user $\mathrm{k}$.

$b_{k}(t)$ is the data sequence for a user $\mathrm{k}$.

$\tau_{k}$ is the delay for user $\mathrm{k}$ relative to a user 0 .

$\varphi_{k}$ is the phase change for user $\mathrm{k}$ relative to a user 0 .

$\omega_{k}$ is the carrier frequency.

In the above equation we assume that there is no multipath interference in the channel. A PN sequence $c_{k}(t)$ is of the form: [10]

$$
\begin{gathered}
c_{k}(t)=\sum_{j=-\infty}^{\infty} \sum_{i=0}^{M-1} a_{k, i} \prod\left(\frac{t-(i+j M) T_{c}}{T_{c}}\right) \\
a_{k, i} \in\{-1.1\}
\end{gathered}
$$

where

$T_{c}$ represents the chip duration.

$M T_{c}$ represents the chip repetition period.

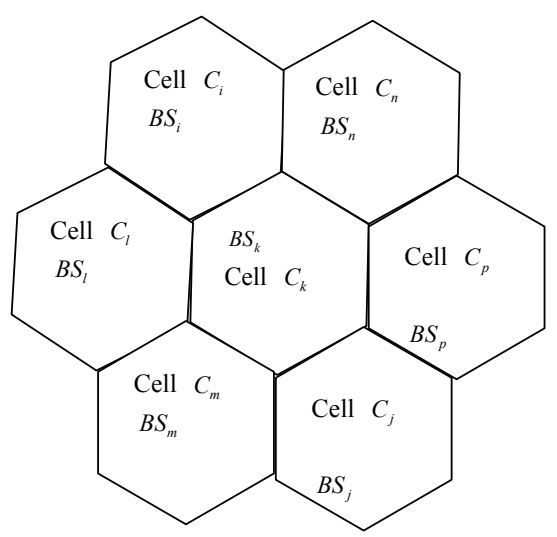

Figure 1. Coverage area.
$\Pi$ represents the unit pulse function given by

$$
\Pi(t)=\left\{\begin{array}{cc}
1 & 0 \leq t \leq 1 \\
0 & \text { otherwise }
\end{array}\right.
$$

$i$ is an index to denote a particular chip within a PN cycle. For data sequence $b_{k}(t), T b$ is the bit period such that

$$
T b=G T c
$$

where $G$ represents the spreading factor or gain of the CDMA system. It is not necessary that the gain $G$ of a CDMA system be equal to $M$. In case they are same, a PN sequence would be repeated for every bit period $T b$.

The user data sequence $b_{k}(\mathrm{t})$ is given by

$$
\begin{gathered}
b_{k}(t)=\sum_{j=-\infty}^{\infty} b_{k, i} \Pi\left(\frac{t-j T_{b}}{T_{b}}\right) \\
b_{k, i} \in\{-1,1\}
\end{gathered}
$$

the signal received at the $\mathrm{BS}$ is given by

$$
r(t)=\sum_{k=0}^{n_{p}} S_{k}\left(t-\tau_{k}\right)+n(t)
$$

where $n(t)$ represents a zero-mean white Gaussian noise with two sided power spectral density $\frac{N_{o}}{2}$, and $n_{p}$ represents the number of users power controlled by BS $\mathrm{p}$.

To derive a decision statistic, the received signal $r(t)$ is mixed with the base band, multiplied with the PN sequence of the desired user, and integrated over the bit period $T_{b}$.

Assuming that the receiver is phase and delay synchronized with the $\mathrm{k}^{\text {th }}$ user, the output of the correlator can be written as $[6,7]$

$$
Z k=\int \begin{gathered}
(j+1) T_{b} \\
j T_{b}
\end{gathered}{ }^{2}(t) c_{k}(t) \cos \left(\omega_{c} t\right) d t
$$

Assume $\tau_{k}=0, \varphi_{k}=0$, and that the desired user is user 0. Hence $k=0$. Substituting Equations (1) and (5) in Equation (6) we obtain

$$
\begin{aligned}
& Z_{0}=\int_{t=0}^{T_{b}}\left[r(t) c_{0}(t) \cos \left(\omega_{c} t\right) d t\right. \\
& =\int_{t=0}^{T_{b}}\left[\left(\sum_{k=0}^{n_{p}-1} \sqrt{2 p_{k}} c_{k}(t-\tau k) b_{k}\left(t-\tau_{k}\right) \cos \left(\omega_{c} t-\varphi_{k}\right)\right.\right. \\
& +n(t)] c_{0}(t) \cos \left(\omega_{c} t\right) d t
\end{aligned}
$$

$\mathrm{Z}_{0}$ is a decision statistic for the desired user.

Equation (7) can be expressed as

$$
Z_{0}=I_{0}+\zeta+\eta
$$

where

1) $I_{0}$ is the contribution from the desired user, i.e.,

$$
I_{0}=\int_{t=0}^{T_{b}} \sqrt{2 P_{k}} b_{0}(t) c_{k}^{2}(t) \cos ^{2}\left(\omega_{c} t\right) d t
$$


As $c_{k} \in\{-1,1\}, c_{k}^{2}=1$

Hence $I_{0}$ reduces to

$$
I_{0}=\sqrt{\frac{P_{0}}{2}} b_{0}(t) T_{b}
$$

2) $\zeta$ represents the contribution of MAI and is the summation of $n_{p}-1$ terms, $I_{k}$, where

$$
\begin{gathered}
I_{k}=\sqrt{2 p_{k}} c_{k}\left(t-\tau_{k}\right) b_{k}\left(t-\tau_{k}\right) \\
\cos \left(\omega_{c} t-\varphi_{k}\right) c_{0}(t) \cos \left(\omega_{c} t\right) d t \\
\zeta=\sum_{k=0}^{n_{p}-1} I_{k}
\end{gathered}
$$

3) $\eta$ represents the contribution of noise and is given by

$$
\left.\eta=\int_{t=0}^{T_{b}} n(t) c_{0}(t) \cos \left(\omega_{c} t\right)\right] d t
$$

To determine the variance and mean of $\eta$

$\mu_{\eta}=E[\eta]=\int_{t=0}^{T_{b}} E\left[n(t) c_{0}(t) \cos \left(\omega_{c} t\right)\right] d t=0$

Variance $\sigma_{\eta}^{2}$

$\sigma_{\eta}^{2}=E\left[\left(\eta-\mu_{\eta}\right)^{2}\right]=E\left[\eta^{2}\right]$

$=\int_{\lambda=0}^{T_{b}} \int_{t=0}^{T_{b}} E\left[n(t) n(\lambda) \cos \left(\omega_{c} \lambda\right) c_{0}(t) \cos \left(\omega_{c} t\right) d t d \lambda\right]$

Now,

$E\left[n(t) n(\lambda)=\frac{N_{0}}{2} \delta(t-\lambda)\right.$

$\sigma_{\eta}^{2}=\int_{\lambda=0}^{T_{b}} \int_{t=0}^{T_{b}} \frac{N_{0}}{2} c_{0}(\lambda) \cos \left(\omega_{c} \lambda\right) c_{0}(t) \cos \left(\omega_{c} t\right) \delta(t-\lambda) d t d \lambda$

$\sigma_{\eta}^{2}=\int_{t=0}^{T_{b}} \frac{N_{0}}{2} c_{0}^{2}(t) \cos ^{2}\left(\omega_{c} t\right) d t$

$c_{0}^{2}(t)=1$

Hence

$$
\begin{gathered}
\sigma_{\eta}^{2}=\int_{\lambda=0}^{T_{b}} \int_{t=0}^{T_{b}} \frac{N_{0}}{4}\left(1+\cos \left(2 \omega_{c} t\right)\right) d t \\
\sigma_{\eta}^{2}=\frac{N_{0} T_{b}}{4}
\end{gathered}
$$

Assuming a large number of interferes, by virtue of the central limit theorem (CLT), the distribution of $\xi$ can be approximated by a zero-mean Gaussian distribution $[4,7,8]$ with variance $\sigma_{\zeta}^{2}$ given by [7]

$$
\sigma_{\zeta}^{2}=\frac{G T_{c}^{2} \sum_{k=1}^{n_{p}-1} P_{k}}{6}
$$

Let,

$$
\xi=\zeta+\eta
$$

Assuming that the MAI and noise are independent proc- esses, the variance of $\xi$ can be written as

$$
\begin{gathered}
\sigma_{\xi}^{2}=\sigma_{\zeta}^{2}+\sigma_{\eta}^{2} \\
=\frac{G T_{c}^{2} \sum_{k=1}^{n_{p}-1} P_{k}}{6}+\frac{N_{0} T_{b}}{4}
\end{gathered}
$$

\subsection{Modeling Intercellular Multiple Access Interference}

Expression for the intercellular interference caused by the users of cell $C_{i}$ at $\mathrm{BS} j$, represented by $I_{i j}$ can be derived. Let the path loss exponent be $m$. Let the fading on path from this user to cell $C_{i}$ be Rayleigh distributed, and represented by $x_{i}$. Similarly, let the fading on the path from this user to cell $C_{j}$ be Rayleigh distributed, and represented by $x_{j}$. The average of $x_{i}^{2}$ is the log-normal fading on the path from this user to cell i, i.e, $E\left[x_{i}^{2} \mid \zeta_{i}=10^{\zeta_{i} / 10}\right.$, where $\zeta_{i}$ is the decibel attenuation due to shadowing, and is a Gaussian random variable with zero-mean and standard deviation $\sigma_{s}$ $[9,10]$. Similarly the average of $x_{j}^{2}$ is the log-normal fading on the path from this user to cell $j$ Let $P_{k}$ be the nominal power received at BS i from user $n_{i}$.It is assumed that the power control mechanism overcomes both the large scale path loss and shadow fading. However, it does not overcome fast fluctuations of signal power due to Rayleigh fading $[8,11]$. As BS i exercises a power control over the MS, the actual transmission power $P_{a c_{i}}$ of the MS would be

$$
P_{a c_{i}}=P_{i}\left[r_{i}(x, y)^{m} 10^{\zeta_{i} / 10}\right]
$$

where $r_{i}(x, y)$ is the distance of the MS from BS $i$. Consequently, assuming uniform user density in the cell, the relative average interference $I_{i j}$ at cell $C_{j}$ caused by all the users in cell $C_{i}$ is given by [9]

$$
I_{i j}=E\left[\frac{n_{i}}{A_{i}} \iint_{c_{i}}^{1} \frac{r_{i}^{m}(x, y) 10^{\zeta_{i} / 10}}{\frac{r_{i}^{m}(x, y)}{x_{j}^{2}}} d A(x, y)\right]
$$

by using iterated expectations,

$$
\begin{gathered}
E\left[10^{\frac{\zeta_{i}}{10}} \cdot x_{j}^{2}\right]=E\left[E\left[10^{\frac{\zeta_{i}}{10}} \cdot x_{j}^{2} \mid \zeta_{i}, \zeta_{j}\right]\right] \\
=E_{\zeta_{i} \zeta_{j}}\left[E\left[10^{\frac{\zeta_{i}}{10}} \cdot x_{j}^{2} \mid \zeta_{i} \zeta_{j}\right]\right]
\end{gathered}
$$

Given $\zeta_{i}$ and $\zeta_{j}$

$$
\left.E\left[x_{j}^{2} \mid \zeta_{i}, \zeta_{j}\right]\right]
$$

is $\log$ normal and is equal to 


$$
10^{-\frac{\zeta_{i}}{10}}
$$

Thus,

$$
E\left[10^{\frac{\zeta_{i}}{10}} \cdot x_{j}^{2}\right]=E\left[10^{\frac{\left(\zeta_{i}-\zeta_{j}\right)}{10}}\right]
$$

Let $X=\zeta_{i}-\zeta_{j}$

Thus, $X$ is a Gaussian variable of zero-mean and variance equal to $2 \sigma_{S}^{2}$

$$
\begin{gathered}
E\left[10^{\frac{\zeta_{i}}{10}} \cdot x_{j}^{2}\right]=E\left[e^{\gamma x}\right] \\
E\left[10^{\frac{\zeta_{i}}{10}} \cdot x_{j}^{2}\right]=\int_{-\infty}^{\infty} \frac{e^{\gamma x} e^{\frac{\gamma x^{2}}{4 \sigma_{S}^{2}}}}{\sqrt{4 \sigma_{S}^{2}}} d x \\
E\left[10^{\frac{\zeta_{i}}{10}} \cdot x_{j}^{2}\right]=e^{\left(\gamma \sigma_{s}\right)^{2}}
\end{gathered}
$$

where $\gamma=\operatorname{In}(10) / 10=.2303$. Substituting the result back in (20) we obtain

$$
I_{i j}=e^{\left(\gamma \sigma_{s}\right)^{2}} \frac{n_{i}}{A_{i}} \iint_{c_{i}}^{1} \frac{r_{i}^{m}(x, y)}{r_{j}^{m}(x, y)} d A(x, y)
$$

If $\alpha$ denotes the voice activity factor, then the above equation becomes

$$
I_{i j}=e^{\left(\gamma \sigma_{s}\right)^{2}} \frac{n_{i} \alpha}{A_{i}} \iint_{c_{i}}^{1} \frac{r_{i}^{m}(x, y)}{r_{j}^{m}(x, y)} d A(x, y)
$$

Let $K_{i j}$ denote inter-cell interference factor due to a user in cell $i$ at $\mathrm{BS} j$.

Hence,

$$
\begin{gathered}
K_{i j}=\frac{I_{i j}}{n_{i}} \\
=e^{\left(\gamma \sigma_{s}\right)^{2}} \frac{\alpha}{A_{i}} \iint_{c_{i}}^{1} \frac{r_{i}^{m}(x, y)}{r_{j}^{m}(x, y)} d A(x, y)
\end{gathered}
$$

In our model $K_{i i}$ is zero at cell ii, but not zero otherwise (i.e, $K_{i j} \neq 0$ ). It is important to point out the importance of $K_{i j} . K_{i j}$ gives the interference at BS $j$ caused by a single user in cell $i$. Thus, if the total number of users in cell $i$ were to change, the new interference levels can be obtained by simply taking a product of $K_{i j}$ and the number of users. This simplifies our calculations as the interference need not be recalculated for the new number of users. Thus, using Equation (23) we can compute relative average intercellular interference for uniform user distribution. Thus, for an uniform user distribution, we can write the total intercellular interference at $\mathrm{BS} j$ due to users in cell $i$ a

$$
I_{i j}=n_{i} \times K_{i j}
$$

It should be noted that the above interference calculations are assuming nominal power as unity. If $P$ is the nominal power from a power controlled user received at home BS, then Equation (26) would be modified as

$$
I_{i j}=p \times n_{i} \times K_{i j}
$$

Equation (27) gives the total intercellular interference at cell $C_{j}$ due to users in cell $C_{i}$.

\section{Simulation and Results}

Simulation was carried out with the following set of fixed parameters:

A chip rate of $1.2288 \mathrm{MHz}$ was selected. Hence, $R c=$ $1.2288 \mathrm{Mcps}$; gain of the CDMA system is 128 . Hence $G=128$; Path loss exponent for mobile communications is 4 [11]. Hence, $m=4$; As we are restricting the interference from the first tier of interferer's, number of cells in the coverage area is 7 . Hence, $N B S=7$; Nominal power of MS, $P k=1$; Speed of radio signal, $\mathrm{C}=3 \times 10^{8} \mathrm{~m} / \mathrm{s}$; Thermal noise, $\frac{N_{0}}{2}=10^{-8}$; User distribution per cell is uniform and every cell has same number of users $N$. The variable sets of parameters include:

a) $N$ : Number of users per cell;

b) $\sigma_{s}$ : The standard deviation of shadowing losses in every cell;

c) $N_{B S}:$ Number of BS's participating in radiolocation;

d) $\Delta:$ DLL(delay-locked loop) resolution.

e) $R$ : Radius of the cells.

We studied the effects of varying shadow losses, varying the number of BS's participating in radiolocation, varying the DLL resolution and varying the size of cells on the accuracy of estimation.

\subsection{Effect of Varying Shadowing Losses on the Accuracy of Radiolocation}

This experiment was conducted to study the combined effect of varying shadowing losses and the number of users per cell on the error in radiolocation.

1) Set $\Delta=1 / 8$, number of BSs participating in radiolocation $=3$, radius of the cell $=1500 \mathrm{~m}$. and integration period, $\mathrm{T}_{\mathrm{int}}=128 \mathrm{~T}_{\mathrm{c}}$. Generate a cell site BS database for 7 cells of radius $1500 \mathrm{~m}$ each, using any computer system that supports the graphical user interface, GUI. Set value of $\sigma_{s}$ to $6 \mathrm{~dB}$.

2) For the given value of $\sigma_{s}$ compute the interference matrix $F_{i j}$.

3) The number of user is vary per cell from 1 to 100 , in steps of 10 , and estimate the error in position estimation

4) Similarly, set $\sigma_{s}=8 \mathrm{~dB}$ and $10 \mathrm{~dB}$, and go to Step 2 . For every setting of $\sigma_{s}$, and number of users per cell, 50 
random locations were chosen within the central cell, and estimations were carried out. The final results are an average of the results obtained at the 50 locations. Similar procedure is carried out for the remaining set of experiments.

The plot in Figure 2 shows the variation of error in radiolocation with number of users per cell and $\sigma_{s}$. The mean value of the radiolocation error, tabulated below is determined by taking an average of all the points plotted in the Figure 2. When the number of users is varied from 1 to 100 , and other conditions remaining the same, the minimum, maximum and mean values of the observed errors are tabulated in Table 1. The mean error in two-dimensional position estimation remains almost constant when $\sigma_{s}$ is increased from $8 \mathrm{~dB}$ to $10 \mathrm{~dB}$.

\subsection{Effect of Varying the Number of Participat- ing BS's on the Accuracy of Radiolocation}

We used 2,3 and 4 BS's to estimate the subscriber location under the above heading. The results are plotted in Figure 3. It was observed that:

1) Accuracy improves drastically if we use more than two BS's for estimation: The accuracy of estimation improves to $71.51 \mathrm{~m}$ from $697.12 \mathrm{~m}$ when we employ $3 \mathrm{BS}$ 's to estimate the subscriber location instead of 2 . Thus, it is very

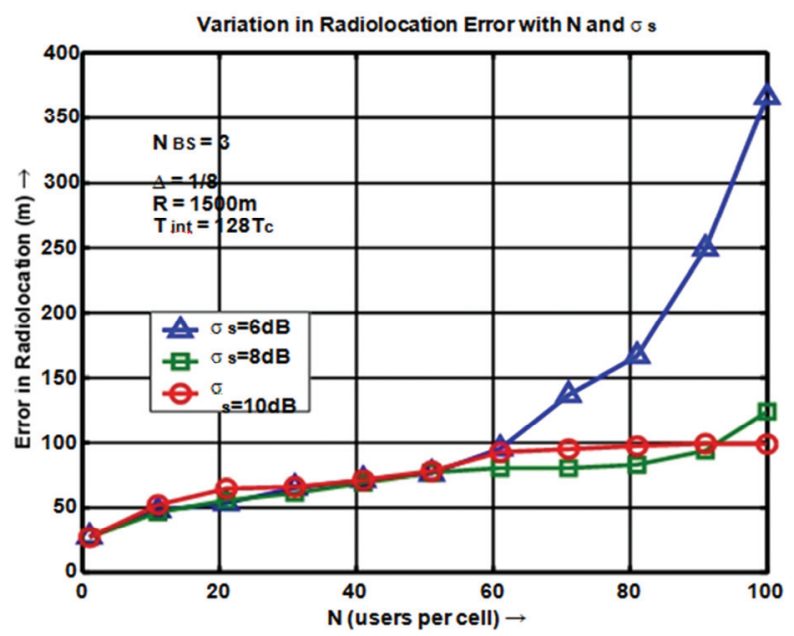

Figure 2. Variation of radiolocation error with $\mathbf{N}$ and $\sigma_{s}$.

Table 1. Min and max error in radiolocation for different values of $\sigma_{s}$

\begin{tabular}{cccc}
\hline $\begin{array}{c}\sigma_{\mathrm{s}} \\
(\mathrm{dB})\end{array}$ & $\begin{array}{c}\text { Minimun } \\
\text { Error } \\
(\mathrm{m})\end{array}$ & $\begin{array}{c}\text { Maximim Error } \\
(\mathrm{m})\end{array}$ & $\begin{array}{c}\text { mean } \\
\text { Error } \\
(\mathrm{m})\end{array}$ \\
\hline 6 & 26.30 & 364.33 & 122.40 \\
8 & 25.83 & 122.30 & 71.51 \\
10 & 25.85 & 96.92 & 75.40 \\
\hline
\end{tabular}

evident that introducing the third estimator has a significant impact on the estimation accuracy and

2) There is no significant improvement in the estimation accuracy when the number of BS's is increased from 3 to 4: The mean radiolocation error improves by $6 \mathrm{~m}$ when we increase the number of BS's to 4. This shows that, there is no effect obtained when we increase the number of BS's from 3 to 4 . For applications with lower accuracy requirements, 3 BS's would be sufficient for radiolocation. Table 2, derived from Figure 3, outlines the minimum, maximum and mean values of estimation errors for various values of $\mathrm{N}_{\mathrm{BS}}$ as the number of users per cell is varied from 1 to 100 , other conditions remaining same.

\subsection{Effect of Varying the Early-Late Discriminator Offset on the Accuracy of Radiolocation}

For our work we have used a non-coherent DLL for estimating the TOA of the received signal. The accuracy of estimating the TOA using a DLL depends on how closely the DLL can track the incoming signal, and this is defined by the parameter $\Delta$. To study the effect of variation of $\Delta$ on the accuracy of estimation, we have performed experiments with $\Delta=\frac{1}{2}, \frac{1}{4}$ and $\frac{1}{8}$. The results of the experiment are plotted in Figure 4.

Table 2. Min and max error in radiolocation for different values of $\mathbf{N}_{B S}$.

\begin{tabular}{cccc}
\hline $\mathrm{N}_{\mathrm{BS}}$ & $\begin{array}{c}\text { Minimun } \\
\text { Error } \\
(\mathrm{m})\end{array}$ & $\begin{array}{c}\text { Maximim } \\
\text { Error } \\
(\mathrm{m})\end{array}$ & $\begin{array}{c}\text { mean } \\
\text { Error } \\
(\mathrm{m})\end{array}$ \\
\hline 2 & 543.10 & 721.64 & 697.12 \\
3 & 26.94 & 24.29 & 71.51 \\
4 & 24.70 & 111.40 & 65.39 \\
\hline
\end{tabular}

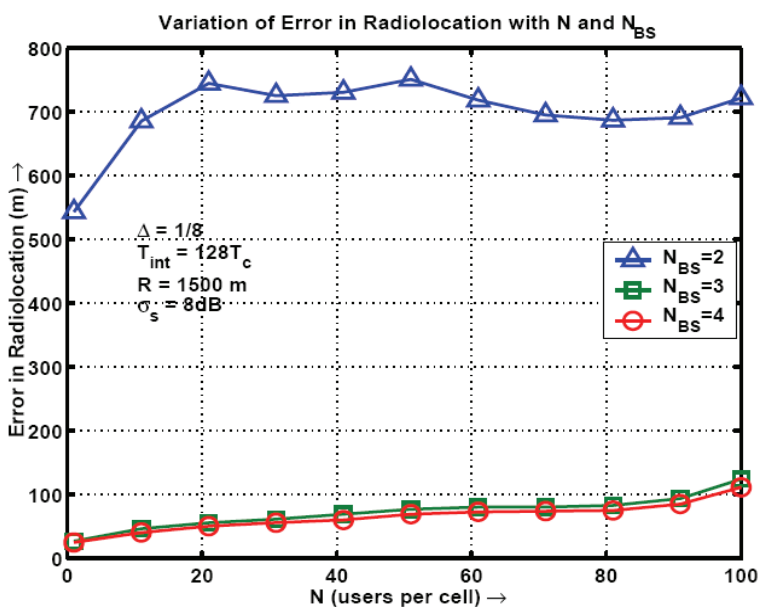

Figure 3. Variation of radiolocation error with $\mathbf{N}$ and $\mathbf{N}_{B S \text {. }}$ 


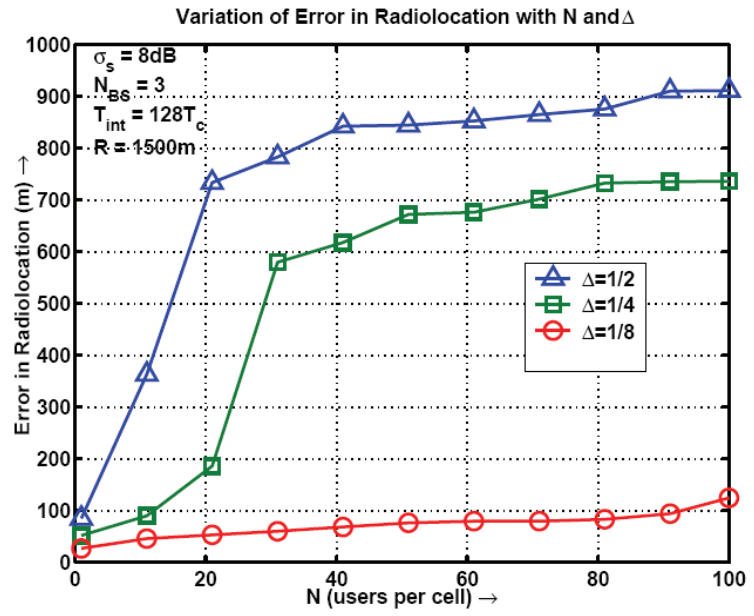

Figure 4. Variation of radiolocation error with $\mathrm{N}$ and $\Delta$.

Table 3, derived from Figure 4, outlines minimum and maximum values of errors for different values of $\Delta$ as the number of users per cell are increased from 1 to 100 , other conditions remaining same. The mean radiolocation error reduces to $524.22 \mathrm{~m}$ and $70.79 \mathrm{~m}$ from $733.13 \mathrm{~m}$ and $524.22 \mathrm{~m}$ respectively when $\Delta$ is reduced from $\frac{1}{2}$ to $\frac{1}{4}$ and from $\frac{1}{4}$ to $\frac{1}{8}$. But, the lowest value of $\Delta$ is limited by

a) In practice, the locally generated $\mathrm{PN}$ sequence will have to be phase delayed to generate the early and late PN sequences. As per IS-95 standards, one chip period corresponds to $813.80 \mathrm{nSec}$. Thus, if we were to deploy a tracking loop with $\Delta=1 / 16$, the requirement on the timing resolution capability on the hardware will be

$$
\Delta \mathrm{t}=\mathrm{T}_{\mathrm{c}} \times \Delta=813.08 \mathrm{nSec} / 16=50.8175 \mathrm{nSec}
$$

Implementing such high precision tracking loops is both challenging and expensive.

b) If the DLL employ's a serial search technique, it will have to search through all potential code delays until the correct delay is identified. Suppose, the incoming $\mathrm{PN}$ sequence is delayed by $\mathrm{T}_{\mathrm{c}}$. If $\Delta=1 / \mathrm{k}$, there are k potential delay values between 0 and $T_{c}$ that the DLL will have to search through before it can lock to the subscriber signal. Thus, the size of the set of potential de-

Table 3. Min and max error in radiolocation for different values of $\Delta$.

\begin{tabular}{cccc}
\hline$\Delta$ & $\begin{array}{c}\text { Minimun } \\
\text { Error } \\
(\mathrm{m})\end{array}$ & $\begin{array}{c}\text { Maximim } \\
\text { Error } \\
(\mathrm{m})\end{array}$ & $\begin{array}{c}\text { mean Error } \\
(\mathrm{m})\end{array}$ \\
\hline $1 / 2$ & 84.86 & 911.02 & 733.13 \\
$1 / 4$ & 51.72 & 735.97 & 524.22 \\
$1 / 8$ & 26.94 & 124.29 & 70.79 \\
\hline
\end{tabular}

lays increases as the value of $\Delta$ decreases. The bigger the set of potential delays, the longer it will take for the tracking loop to achieve a lock. The situation becomes more complicated, if we are also estimating the velocity of the subscriber. The set of potential delays, soon transforms into a two-dimensional matrix defining the set of potential delays and velocities. A serial search technique would be inefficient for such cases.

Also, accuracy falls as number of users per cell increases. As seen in Figure 4, the accuracy of estimation, falls as the number of users per cell increases. This is because of the degradation of SNR (signal-to-noise) with increasing number of users per cell.

\subsection{Effect of Varying the Cell Size on the Accuracy of Radiolocation}

All the earlier experiments were carried out with cells, each of radius $1500 \mathrm{~m}$. In this case, we simulated coverage areas with cell radii $100 \mathrm{~m}$ and $500 \mathrm{~m}$. Simulations were carried out under the following conditions: $\Delta=1 / 8 ; \sigma_{\mathrm{s}}=$ $8 \mathrm{~dB}$; and number of BSs involved in radiolocation $=3$. The number of users is varied from 1 to 100 in steps of 10 .

The results were then compared with the results of the experiment carried out under identical situation but using cells of $1500 \mathrm{~m}$ radius. The results indicate that under perfect power control, the degradation in SNR (signal-to-noise) with number of users is independent of the cell size; and the accuracy of estimation is better with smaller cells.

For the experiment conducted with cells of radii $100 \mathrm{~m}$, $500 \mathrm{~m}$ and $1500 \mathrm{~m}$, it is found that accuracy of radiolocation is best for cells of radius 100 .

\section{Conclusions}

Our study has investigated the possibility of accurate subscriber location in CDMA cellular networks in the presence of multiple access interference. Earlier works have ignored the effect of non-orthogonality of the pseudo-noise codes on the estimation accuracy. They usually consider the case of a single MS with no interferers, which is not a practical assumption. In our work, we have studied the effect of number of interferers on the accuracy of estimation by varying the number of users in every cell from 1 to 100 . To study the effects of multiple access interference on the accuracy of estimation, we have assumed the presence of a line-of-sight component between the MS and the BS. We have studied the effect of MAI in conjunction with varying shadowing environments, varying tracking capability of the DLL, varying number of BSs participating in radiolocation, and varying cell sizes. The results obtained through the simulations carried out under these different conditions are encouraging and show that radiolocation is possible in a CDMA system, even when multiple access interfer- 
ence is present.

\section{References}

[1] R. Ilts, "Joint estimation of PN code delay and multipath using extended kalman filter," IEEE Transactions on Communications, Vol. 38, pp. 1677-1685, October 1990.

[2] M. Silventoinen and T. Rantalainen, "Mobile station emergency locating in GSM," Proceeding of IEEE International Conference on Wireless Communications, pp. 232-38, 1996.

[3] M. Wyile and J. Holtzman, "The non line of sight problem in mobile location estimation," Proceedings of IEEE ICUPC, pp. 827-831, 1996.

[4] J. Caffery, Jr, and G. Stuber, "Subscriber location in CDMA cellular networks," IEEE transactions vehicle technology, Vol. 47 No. 2, pp. 406-15, May 1998.

[5] M. Hata and T. Nagatsu, "Mobile location using signal strength measurements in a cellular system," IEEE Transactions Vehicle Technology, Vol. 29, pp. 245251, May 1980.
[6] R. K Morrow, Jr., and J. S. Lehnert, "Bit-to-bit error dependence in slotted DS/SSMA packet systems with random signature sequences," IEEE Transactions on Communications, Vol. 37, No. 10, October 1989.

[7] T. S. Rappaport, "Wireless communications principles \& practice," IEEE Press, New York, Prentice-Hall, 2002.

[8] A. J. Bamisaye and M. O. Kolawole, "Evaluation of downlink performance of a multiple-cell, rake receiver assisted CDMA mobile system," Journal of Wireless Sensor Network, 2009.

[9] R. G. Akl, M. V. Hegde, A. Chandra, and P. S. Min, "CCAP: CDMA capacity allocation and planning," Tech Republic, Washington University, April 1998.

[10] W. C. Y. Lee, "Mobile cellular telecommunications, analog and digital systems," 2nd Edtion, McGraw-Hill, Inc, 1990.

[11] K. Krizman, T. Biedka, and T. Rappaport, "Wireless position location: Fundamentals implementation strategies, and sources of error," in Proceedings of Vehicular Technology Conference, pp. 919-923, 1997. 